\title{
Medical Emergencies in Dentistry: A Review
}

\author{
Jyoti Zingade ${ }^{1}$, Garish Kumar ${ }^{2}$, Pavan K Gujjar ${ }^{3}$
}

\begin{abstract}
Medical emergencies have a way of happening unexpectedly, so one has to be prepared for an emergency before it happens. When a true emergency happens in the hospitals, clinics, or in home, the first few minutes can mean the difference between life and death. If we review protocol thoroughly before the stress of an emergency, we will be better able to think with a clear head. This will enable to react effectively and quickly to provide help to someone who really needs it. When the dentist sets priorities for preventive measures, an understanding of the relative frequency of emergencies and knowledge of those likely to produce serious morbidity and mortality are important. Recognition, prevention, preparation, basic life support, cardiopulmonary resuscitation, and specific medical emergencies are the priorities for the prevention of medical emergencies in dentistry. This review gives the idea of common medical emergencies, which may occur in dental office and their management protocol.

Keywords: Cardiopulmonary Resuscitation, Emergency, Shock, Syncope.

Journal of Health Sciences \& Research (2021): 10.5005/jp-journals-10042-1103
\end{abstract}

\section{INTRODUCTION}

Any adverse medical condition arising in the dental practice, which is not corrected promptly, may progress to life-threatening or incapacitating consequences that are termed as medical emergencies. It can and does happen due to advances in medicine, longer life span, multiple medications, and medically compromised, longer appointments. It is observed that, over a period of 10 years, $90 \%$ of dentists have encountered at least one medical emergency (Table 1). ${ }^{2}$

\section{UNCONSCIOUSNESS}

It is also called as syncope, or faint means sudden, transient loss of consciousness. Predisposing factors: It is mainly due to three factors, namely, stress, impaired physical status, and administration or ingestion of drugs like analgesics, antianxiety agents, and antibiotics.

Possible causes of unconsciousness in dental office are vasodepressor syncope, drug administration/ingestion, orthostatic hypotension, epilepsy, hypoglycemic reaction, acute adrenal insufficiency, acute allergic reaction, acute myocardial infarction, hyperglycemic reaction, and hyperventilation syndrome. ${ }^{3}$

The most common cause for the loss of consciousness in the dental office is simple fainting under stress. A medically compromised patient will have a more serious reaction to stress (both psychological and physiological) than a healthy counterpart undergoing the same procedure. Most drugs used in dental treatment are central nervous system depressants and have the potential to induce the loss of consciousness. ${ }^{4}$ The most common time of occurrence of vasodepressor syncope is during or immediately following the dental local anesthetic injection.

\section{Vasodepressor Syncope}

It is caused by a decrease in cerebral blood flow below a critical level and is usually characterized by a sudden fall in blood pressure and slowing of the heart rate. ${ }^{5}$

This generally occurs because of a series of cardiovascular events triggered by the emotional stress brought on during
${ }^{1}$ Department of Oral Medicine and Radiology, MNDAV Dental College and Hospital, Solan, Himachal Pradesh, India

2,3 Department of Oral Pathology and Microbiology, MNDAV Dental College and Hospital, Solan, Himachal Pradesh, India

Corresponding Author: Pavan K Gujjar, Department of Oral Pathology and Microbiology, MNDAV Dental College and Hospital, Solan, Himachal Pradesh, India, Phone: +91 8347060388, e-mail: jyopavan2612@gmail.com

How to cite this article: Zingade J, Kumar G, Gujjar PK. Medical Emergencies in Dentistry: A Review. J Health Sci Res 2021;12(1):11-16. Source of support: Nil

Conflict of interest: None

the dental procedures. The initial event in episode is the stressinduced release of increased amounts of catecholamines that cause a decrease in peripheral vascular resistance, tachycardia, and sweating. The patient may complain of feeling generalized warmth, nausea, and palpitations. ${ }^{6}$ As blood pools in the periphery, a drop in the arterial blood pressure appears, which leads to a decrease in cerebral blood flow. The patient may then complain of feeling dizzy or weak. Once the blood pressure drops below levels necessary to sustain consciousness, syncope occurs even the compensatory mechanisms attempt to maintain adequate blood pressure as they soon fade, leading to vagally mediated bradycardia.

Factors that can precipitate vasodepressor syncope may be divided into two groups:

1. Psychogenic that includes fright, anxiety, emotional stress, pain, sight of blood, sight of surgical instruments.

2. Non-psychogenic includes upright or standing position, hunger, exhaustion, hot, humid environment.

\section{Clinical Manifestation}

- Presyncope: Feeling of warmth in face and neck, loss of color: pale, heavy perspiration, nausea, rapid heart rate, BP depressed.

(0) The Author(s). 2021 Open Access This article is distributed under the terms of the Creative Commons Attribution 4.0 International License (https:// creativecommons.org/licenses/by-nc/4.0/), which permits unrestricted use, distribution, and non-commercial reproduction in any medium, provided you give appropriate credit to the original author(s) and the source, provide a link to the Creative Commons license, and indicate if changes were made. The Creative Commons Public Domain Dedication waiver (http://creativecommons.org/publicdomain/zero/1.0/) applies to the data made available in this article, unless otherwise stated. 
Table 1: Classification of emergencies

\begin{tabular}{ll}
\hline Non-cardiovascular & Cardiovascular \\
\hline A. Stress-related & A. Stress-related \\
Vasodepressor syncope & Heart failure \\
Hyperventilation syndrome & $\begin{array}{l}\text { Cerebral ischemia and infraction } \\
\text { Hypertensive crisis } \\
\text { Asthma adrenal insufficiency }\end{array}$ \\
$\begin{array}{l}\text { Hypoglycemic reactions } \\
\text { Seizures }\end{array}$ & \\
$\begin{array}{l}\text { B. Non-stress-related } \\
\text { Orthostatic hypotension } \\
\text { Overdose reaction } \\
\text { Hyperglycemia }\end{array}$ & B. Non-stress-related \\
Anaphylactic reactions & Acute myocardial infraction \\
\hline
\end{tabular}

\begin{tabular}{|c|c|c|}
\hline Step & Action & Comment \\
\hline Step 1 & $\mathrm{P}$ (position) & $\begin{array}{l}\text { As soon as symptoms appear-halt } \\
\text { the procedure } \\
\text { supine position with legs slightly } \\
\text { elevated (Trendelenburg position- } \\
\text { B.P. increases by } 2 \mathrm{~mm} \mathrm{Hg} \text { for each } \\
\text { inch below heart) }\end{array}$ \\
\hline Step 2 & $\begin{array}{l}\text { ABC (airway, } \\
\text { breathing, and } \\
\text { circulation) }\end{array}$ & $\begin{array}{l}\mathrm{O}_{2} \text { or ammonia ampule under the } \\
\text { nose } \\
\text { (stimulates breathing and muscular } \\
\text { movement) }\end{array}$ \\
\hline Step 3 & Definitive care & $\begin{array}{l}\text { Determine the cause of syncope } \\
\text { Modification in further dental treatment } \\
\text { to minimize the risk }\end{array}$ \\
\hline Step 4 & $\begin{array}{l}\text { Administration } \\
\text { of oxygen }\end{array}$ & At any time during episode \\
\hline Step 5 & $\begin{array}{l}\text { Monitoring of } \\
\text { vital signs }\end{array}$ & \\
\hline \multicolumn{3}{|c|}{$\begin{array}{l}\text { Step 6: Additional procedures-loosening of binding clothes, } \\
\text { using respiratory stimulant (ammonia), blanket if patient shivering, } \\
\text { bradycardia-anticholinergics, removal of instruments, or stimulus } \\
\text { which precipitated the episode. }\end{array}$} \\
\hline
\end{tabular}

- Syncope: Hyperpnoea, hypotension, bradycardia, visual disturbances, dizziness, convulsive movement and muscular twitching, loss of consciousness.

- Post-syncope (recovery) : Exhibit pallor, nausea, weakness and sweating, mental confusion, or disorientation.

\section{Management}

Preventive reactions involve proper patient preparation. The extremely anxious patient should be treated by using an anxiety reduction protocol and, if necessary, pretreatment anxiolytic drugs can be given. The following steps should be followed to recover the patient ${ }^{7}$ :

\section{Syncope in Pregnant Patients}

\section{Supine Position}

Supine position decreased venous return to heart-decreased blood supply to the brain. In such cases, supine positioning may actually lead to a decrease in the return of venous blood to the heart. The gravid uterus may obstruct or diminish the blood flow through the inferior vena cava on the right side of the abdomen trapping large amount of blood in the legs. ${ }^{8}$

\section{Management}

One situation requiring modification of this basic positioning is the loss of consciousness in a pregnant woman near term. Turn the patient to her right side, and tuck a pillow or blanket under the left side of her back to help her maintain that position.

\section{Orthostatic Hypotension (Postural Hypotension)}

This is the second most common cause of loss of consciousness. It is not usually related to anxiety like vasodepressor syncope but it is commonly caused by sitting upright or standing, which leads to a drop in blood pressure in some patients. Standing systolic blood pressure drops at least $25 \mathrm{~mm} \mathrm{Hg}$, and standing diastolic blood pressure drops at least $10 \mathrm{~mm} \mathrm{Hg}$. This problem occurs because of pooling of blood in the periphery that is not remobilized quickly enough to prevent cerebral ischemia, when a patient rapidly assumes an upright posture. The patient will therefore feel lightheaded or become syncopal. Patients who remain conscious will usually complain of palpitations and generalized weakness. ${ }^{9}$

The elderly, pregnant, patients reclining for a long time, patients with Addison's disease, and those medicated with nitrous oxide and oxygen, diazepam, or some drugs used in IV sedation are at a greater risk for orthostatic hypotension. The patients are usually asymptomatic just before the incident and return to consciousness after lying down. ${ }^{10}$

\section{Management}

- Terminate all types of dental treatments.

- Supine positioning of the patients, with legs raised above the level of the head.

- Monitor vital signs like pulse, respiratory rate, and blood pressure.

- Once blood pressure improves, slowly return patient to sitting posture.

- Discharge to home once vital signs are normal and stable.

- Obtain medical consent before any further dental care.

\section{Seizures}

It is a paroxysmal disorder of cerebral function characterized by an attack involving changes in the state of consciousness. Idiopathic seizure disorders are exhibited in many ways, ranging from grand mal seizures to petit mal seizures that may occur with only episodic absences (e.g., blank stare).

Seizures or convulsions are caused by a disturbance in the electrical activity in the brain that leads to a series of uncontrolled muscle movements. The patient may be totally or partially unconscious with a temporary breathlessness. The episode usually lasts 1 or 2 minutes. There are certain causes of seizures like head injury, brain tumor, epilepsy, poisoning, electric shock, withdrawal from drugs, heat stroke, scorpion or poisonous snakebites, hyperventilation, or high fever. ${ }^{11}$

Predisposing factors include hypoxia, metabolic disorders, congenital deformity, birth injury, toxin, trauma, and cerebral degenerative diseases.

\section{Clinical Manifestation}

Expression of a short cry or scream, muscles become rigid, jerky twitching movements, temporary interruption of breathing, face and lips turn bluish, eyes roll up into the patient's head, loss of bladder and bowel control, drooling and unresponsiveness during the seizure. The patient may feel sleepy and confused following the episode. ${ }^{12}$ 
Absence seizure occurs in children of 3 to 15 years of age. Complete suppression of all mental function, blank stare, sudden immobility, intermittent blinking at three cycles per second, and it lasts for 5 to 30 seconds.

\section{Tonic-clonic Seizure}

Tonic: sustained generalized skeletal muscle contraction occurs first in flexion and then progresses to tonic extensor rigidity of the extremities and trunk; dyspnea and cyanosis may be evident; it lasts for 10 to 20 seconds.

Clonic: muscular relaxation, violent, vigorous movements seen, tongue bite may occur; it lasts for 2 to 5 minutes.

- Prodromal phase: Anxiety, depression, aura-first sign of disease, postpone the treatment.

- Preictal phase: Loses consciousness, falls on floor, increases heart rate, blood pressure.

- Ictal phase: Serious generalized skeletal muscle contractions, tonic extensor rigidity clonic movements of body, bite the tongue.

- Postictal phase: Returns to normal.

Diagnostic clues include sudden onset of immobility, blank stare, and slow blinking of eyelids.

Management: The best thing to do during an epileptic episode is to take the patient at safe place on the ground so he or she doesn't injure himself or herself and let the convulsion run its course. Do not put anything in the patient's mouth, and do not try to hold them. After the convulsion has subsided, check the patient's respiration. ${ }^{7}$ If breathing does not start soon after the seizure is over, reposition the patient's head to open their airway. Check that the tongue has not blocked the back of the throat. As soon as breathing returns to a normal pace, roll the patient on their side to avoid choking by any secretions. ${ }^{13}$

\section{Status Epilepticus}

Continuous or repeated seizures without any periods of recovery are known as status epilepticus. This problem affirms the notification of outside emergency assistance because it is the most common type of seizure disorder to cause mortality.

Therapy includes initial measures that should start for selflimiting seizures; in addition, the administration of a benzodiazepine is indicated.

- However, the doctor administering benzodiazepines for a seizure must be prepared to provide basic life support (BLS), because patients may experience a period of apnea after receiving a large and rapid dose of benzodiazepines.

- Diazepam-5 to $10 \mathrm{mg}$ at $5 \mathrm{mg} /$ minutes repeated every 10 minutes till $30 \mathrm{mg}$ maximum dose.

- Phenytoin (20 mg/kg IV)_for long-term seizures.

- Phenobarbital (20 mg/kg IV)—for continuing seizures.

\section{Allergy}

It is defined as a hypersensitive state acquired through exposure to a particular allergen, and re-exposure to which produces a heightened capacity to react. Hypersensitivity (or allergy) literally defines an exaggerated response of the immune system to antigenic challenge, harmful to the organism itself. It is the intolerance of the body to a specific substance due to an immune response to a specific antigen. Common allergies include asthma, hay fever, and eczema. ${ }^{14}$

Anaphylactic Shock: Anaphylaxis $\rightarrow$ Ana-without, phylaxisprotection

It is the most serious and life-threatening allergic reaction. The body's vital functions like breathing and circulation are impaired, and oxygen cannot reach the brain.

There are four basic types of hypersensitivity reactions; among those, type I (immediate hypersensitivity) can cause an acute, life-threatening condition. Type I allergic reactions are mediated primarily by immunoglobulin $\mathrm{E}$ ( $\mathrm{IgE}$ ) antibodies. Initiation of a type I response requires exposure to an antigen previously seen by the immune system. The re-exposure to the antigen triggers a cascade of events that then are exhibited locally, systemically, or both in varying degrees of severity. ${ }^{15}$

\section{Clinical Manifestation}

The most common and severe manifestation of type 1 hypersensitivity is dermatologic. Skin or mucosal reactions include localized areas of pruritus, erythema, urticaria, and angioedema. Although skin and mucosal reactions are not much dangerous, it may be the first indication of more serious allergic manifestations that will soon follow.

Allergic reactions affecting the respiratory tract are more serious and require more aggressive intervention. The involvement of small airways occurs with wheezing, as the constriction of bronchial smooth muscle (bronchospasm) and airway mucosal inflammation occurs. The patient will complain of dyspnea and may eventually become cyanotic. ${ }^{16}$

Involvement of the larger airways usually first occurs at the narrowest portion of those air passages - the vocal cords in the larynx. Angioedema of the vocal cords causes partial or total airway obstruction. Nausea and vomiting, abdominal cramping, and urinary incontinence may occur. Symptoms of respiratory embarrassment soon follow, with dyspnea and wheezing. Cyanosis of nail beds and mucosa will next appear if air exchange becomes insufficient. Finally, total airway obstruction occurs, which causes the patient to quickly become unconscious. ${ }^{17}$

\section{Management}

- The patient should be positioned supine, and medical assistance should be given immediately.

- For patients with milder symptoms like rash or itching, he or she should be given diphenhydramine 50 to $100 \mathrm{mg}$ IV or IM, and vital signs should be monitored.

- A preloaded syringe of epinephrine should be given in case it is needed.

- Monitor vital signs like pulse, blood pressure, and respiratory rate.

\section{Local Anesthesia Overdose Toxicity}

"Administer Less Than Maximum Cartridges Allowed or Less Than Maximum Dosage Allowed, Whichever Is Less. Always take the weight of the patient into account."

Local anesthesia (LA) is linked to $50 \%$ of the deaths in the dental office. Use the smallest dose that will produce adequate anesthesia, which is mandatory. Toxicity level increases by administering too much of the drug (especially as related to the patient's body weight), administering the drug to a sensitive individual, administering the drug into a blood vessel, or by 
improper drug combinations. If the level of the LA is too high, it can become toxic causing a dangerous reaction in the nervous system, in cardiovascular system, or in the local tissues. The rate of absorption and elimination of the drug is directly related to its toxic effects. The faster it is absorbed by the bloodstream and the slower it is metabolized, the more toxic it is to the body. ${ }^{18}$

\section{Clinical Manifestations}

The most commonly occurring symptoms are slurred speech, excitement, shivering, muscular twitching, and tremor of facial muscles and extremities. The patient may also feel numbness of the tongue, warm, flushed skin, lightheadedness, dizziness, diminished sight, tinnitus, and disorientation.

\section{Prevention of LA Toxicity}

- The amount of LA dose used should be determined based on the necessity of the treatment, which produces the intensity and duration of pain control required to successfully complete the planned surgical procedure.

- While choosing the dose of LA, the patient's age, lean body mass, liver function, and history of problems with LAs must be considered.

- Method of drug administration, the second most important factor, to consider in preventing a LA overdose reaction.

- The dentist should give the required dose slowly, avoid intravascular injection, and use vasoconstrictors to slow the entry of LAs into the blood.

- The choice of LA agents is the third important factor to consider in attempting to lessen the risk of a toxicity reaction. Therefore, the dentist must be knowledgeable about the various LAs available and its doses.

\section{Management}

- Position patient (supine)

- Prevent injury, and loosen tightened garments

- Record vital signs

- BLS

- Anticonvulsant after 4 to 5 minutes, diazepam 2 mg/minutes IV

- Additional management, cardiopulmonary resuscitation (CPR) if indicated

\section{Asthma}

It is defined as a clinical state of hyper-reactivity of the tracheobronchial tree, characterized by recurrent paroxysms of dyspnea and wheezing. Four types ${ }^{14}$ :

1. Extrinsic asthma: Allergic asthma: $50 \%$ the allergens may be airborne such as house dust, feathers, fungal spores, plant pollens, food (cow's milk, eggs, fish tomatoes) and drugs (penicillins, aspirin sulfites): bronchospasm occurs, acute attacks of extrinsic asthma may occur. Fifty percentage of children are asymptomatic till adulthood

2. Intrinsic asthma: Common in older than in 35 years: nonallergic factors such as respiratory infection, physical exertion, environmental and air pollution, occupational stimuli

3. Mixed asthma: Combination of intrinsic and extrinsic

4. Status asthmaticus: More severe, experiences wheezing, dyspnea, hypoxia

\section{Precautions to be taken}

- If a patient were experiencing asthma attacks due to emotional stress, he or she would benefit from a stress reduction exercise before beginning the treatment (especially if he or she indicates a fear of dentistry).

- Use of nitrous oxide may be indicated as non-irritating to the respiratory mucosa and has an excellent calming effect.

- Some asthmatics are claustrophobic; in those cases, nasal cannula may be used instead of a nosepiece.

- Asthmatic patients should be reminded to bring their medication with them to all dental appointments.

- Aspirin and other nonsteroidal anti-inflammatory drugs or penicillin are contraindicated in asthmatics, because they might trigger asthmatic attacks. Any anesthetic containing bisulfite as a preservative is also contraindicated in an asthmatic patient.

\section{Clinical Manifestations}

Difficulty in exhalation, wheezing or whistling, anxiety, nervousness, coughing, perspiration on forehead, choking sensation, possible vomiting or fever, and bluish tinge to skin are the most common signs. $^{10}$

\section{Management}

- The patient should sit upright (erect/semi-erect) because it is easier to breathe in this position.

- If the patient is on medication, then he/she should take it according to the prescribed directions.

- Patients should then administer bronchodilators, using their own inhalers or one provided from the office emergency supply. The inhaler may contain epinephrine, isoproterenol, metaproterenol, or albuterol.

- Oxygen administration should follow, using nasal prongs or a face mask.

- In more severe asthmatic episodes or when aerosol therapy is ineffective, epinephrine $(0.3 \mathrm{~mL}$ of a 1:1000 dilution) may be injected SC or IM, hydrocortisone succinate, 100 to $200 \mathrm{mg}$ IV.

- When patients have severe respiratory embarrassment, it may be necessary to obtain outside emergency medical assistance.

\section{Hyperventilation}

It is defined as ventilation in excess of that required to maintain normal blood $\mathrm{PaO}_{2}$ and $\mathrm{PaCO}_{2}$. Hyperventilation is a fairly common emergency in the dental office.

A patient may hyperventilate due to extreme anxiety, pain, metabolic acidosis, drug use, hypercapnia, cirrhosis, and some central nervous system disorders. The best prevention for hyperventilation is to address any anxieties about dentistry before starting treatment. ${ }^{19}$

\section{Clinical Manifestations}

The first manifestation of hyperventilation syndrome is patient complains of an inability to get enough air. Lightheadedness, numbness or tingling in the extremities and around the mouth and lips, muscle twitches, and difficulty in catching a deep breath are the major symptoms. The patient usually does not lose consciousness, but prolonged hyperventilation may lead to convulsions.

They breathe very rapidly and become agitated. The rapid ventilation increases the elimination of $\mathrm{CO}_{2}$ through the lungs. The patient rapidly becomes alkalotic; may complain of becoming lightheaded and of having a tingling sensation in the 
fingers, toes, and perioral region; and may even develop muscle twitches or convulsions. Eventually, loss of consciousness occurs.

\section{Management}

- Management of a hyperventilating patient involves terminating the surgical procedure, positioning the patient in a semi-erect position, and providing reassurance.

- If symptoms of alkalosis occur, the patient should be forced to breathe into and out of a small bag.

- Oxygen-rich air is not indicated.

- If hyperventilation continues, the clinician may have to administer a sedative such as midazolam, by giving 2 to $4 \mathrm{mg}$ IM or by IV titration of the drug until hyperventilation ceases or the patient is sedated.

\section{Diabetes Mellitus}

Diabetes mellitus is a group of metabolic disorders characterized by chronic hyperglycemia due to relative insulin deficiency.

Predisposing factors: Genetic predisposition, primary destruction of islets of Langerhans in the pancreas caused by inflammation, cancer or surgery, endocrine condition such as hyperpituitarism or hyperthyroidism, administration of steroids.

Two main clinical entities of DM are hyperglycemia and hypoglycemia

\section{Hyperglycemia}

It may be precipitated by the following factors, all of which increase body's requirements for insulin: weight gain, pregnancy, hyperthyroidism, thyroid medications, epinephrine therapy, corticosteroid therapy, acute infection. ${ }^{20}$

Management: In clinic if patient becomes unconscious, take the following measures: Establish IV line and administer $0.9 \%$ saline, $1 \mathrm{~L}$ in 30 minutes $+\mathrm{KCl} 20$ mmol.

\section{Hypoglycemia}

The second cause of the acute complication of diabetes mellitus, hypoglycemia, may rapidly progress to the loss of consciousness. Hunger, nausea, mood change, weakness, tachycardia, perspiration, pallor, anxiety, behavioral change, hypotension, unconsciousness, and seizures.

\section{Management}

- Administer oral glucose source 20 to $40 \mathrm{mg}$

- Monitor vital signs

- IV D50\%, $50 \mathrm{~mL}$ or glucagon $1 \mathrm{mg}$

- Give $\mathrm{O}_{2}$

- Consult physician

\section{Respiratory Difficulty}

Possible causes of respiratory difficulty are hyperventilation syndrome, vasodepressor syncope, asthma, heart failure, hypoglycemia, overdose reaction, acute myocardial infarction, anaphylaxis, angioneurotic edema, epilepsy, and hyperglycemic reaction

Airway obstruction in dental office are inlays, burs, mouth mirror heads, (gold) crowns, pieces of debris, rubber dam clamps, endodontic instruments, post and core that may fall into the oropharynx of the patient. Swallowed object entering GIT, objects aspirated into bronchus.

\section{Clinical Manifestations}

Inability to speak, inability to breath, inability to cough, universal sign for choking, panic.

Signs of partial airway obstruction with good air flow, forceful cough, wheezing between coughs, ability to breathe. With poor air flow, weak, ineffectual cough, crowing sound on inspiration, altered voice sounds, possible cyanosis, and lethargy. ${ }^{21}$

\section{Management}

Partial obstruction: If patient capable of forceful coughing.

\section{Total Airway Obstruction}

Back blows-integral part, patient is straddled over the rescuer's arm, with the head lower than the trunk and the head supported by firmly holding the jaw.

\section{Manual thrusts}

Heimlich maneuvers (abdominal thrust): Repeat inward and upward thrusts until either the foreign body is expelled or the victim loses consciousness

Chest thrust: They function to rapidly increase the intrathoracic pressure, acting as an artificial cough that may help to dislodge a foreign body

Prevention of airway obstruction in dental office by using Rubber dam, oral packing, chair position, dental assistant, Magill intubation forceps, ligature.

\section{Adrenal Insufficiency}

Primary adrenocortical insufficiency (Addison's disease) or other medical conditions in which the adrenal cortex has been destroyed are rare.

However, adrenal insufficiency secondary to exogenous corticosteroid administration is relatively common because of the multitude of clinical conditions for which therapeutic corticosteroids are given. Patients with adrenal insufficiency are frequently not informed concerning their potential need for supplemental medication, and those with secondary adrenal insufficiency may fail to inform the dentist that they are taking corticosteroids.

Patients at risk of acute adrenal insufficiency as a result of adrenal suppression are generally those who take at least $20 \mathrm{mg}$ of cortisol (or its equivalent) daily for at least 2 weeks any time during the year preceding the planned major oral surgical procedure.

\section{Clinical Manifestations}

Mental confusion, nausea, fatigue, and muscle weakness. As the condition worsens, the patient develops more severe mental confusion; pain in the back, abdomen, and legs; vomiting; and hypotension. Without treatment, the patient will eventually begin to drift in and out of consciousness, with coma harkening the preterminal stage. ${ }^{22}$

\section{Management}

- Stop all dental treatment and taking vital signs.

- If the patient is found to be hypotensive, they must be immediately placed in a head-down, legs elevated position.

- Medical assistance should be summoned.

- Oxygen should be administered and venous access gained.

- A 100-mg dose of hydrocortisone sodium succinate should be given IV (or IM, if necessary). IV fluids are rapidly administered until hypotension improves.

- Monitor vital signs 


\section{Heart Failure}

When there is a failure in supply the oxygenated blood by the heart to the body for normal functioning, it is referred to as heart failure. Patients who report history of heart failure are at an increased risk during dental treatment. The patient health history should be reviewed thoroughly for any heart disease that may have weakened the heart muscle, and current circulatory and heart conditions that may predispose a patient to congestive heart failure. A physical evaluation of the patient should be considered as well. Note the vital signs at the initial appointment, and compare them to the present signs.

\section{Clinical Manifestations}

Cold and pale skin; sweating, pitting edema of the ankles; fatigue, dyspnea on exertion; hyperventilation; and wheezing sound, cyanosis, frothy pink sputum, increased anxiety, and dyspnea at rest.

\section{Management}

- The treatment should be conducted in a stress-free environment.

- If the patient exhibits anxiety about dental treatment, consider oral sedatives, nitrous oxide oxygen, or intravenous sedation.

- The patient should be instructed to take his or her medication regularly, especially the days before and the day after the treatment.

- The dentist must consider the medications being taken by a hypertensive patient and how they may interact with the medications for dental treatment. ${ }^{20}$

\section{Conclusion}

Basic preparation of the office for all emergency situations is critical to the successful management of emergencies in dental office. The development of life-threatening situations can be minimized using certain factors like pretreatment physical evaluation of each patient, which consists of a medical history questionnaire, dialogue history and physical examination, and possible modifications in dental care to minimize medical risks.

Medical emergencies can happen anywhere. Dental visit and treatments can trigger an emergency in sensitive patients. Knowledge is power so know what to do, know your limitations, and most of all, know when to call the experts.

\section{References}

1. General Dental Council. Scope of practice. 2013. Available at: https:// www.gdc-uk.org/docs/default-source/scope-of-practice/scope-ofpractice.pdf.
2. Al-Turki O, Al-Hussyeen A, Al-Hammad N, et al. Medical emergencies in dental practice. J Dent Med Sci 2017;16:1-9. DOI: 10.9790/08531612080109.

3. Greenwood M, Meechan J. General medicine and surgery for dental practitioners: Part 2. Medical emergencies in dental practice: the drug box, equipment and basic principles of management. Br Dent J 2014;216(11):633-637. DOI: 10.1038/sj.bdj.2014.447.

4. Jevon P. New poster to help manage medical emergencies. Br Dent J 2009;207:312. DOI: 10.1038/s41415-020-1789-y.

5. Medicines and Healthcare Products Regulatory Agency. Drug Safety Update 2019;13(3). Available at: https://assets.publishing.service. gov.uk/government/uploads/system/uploads/attachment_data/ file/840565/oct-2019-pdf.pdf.

6. Ewan $\mathrm{P}$, Brathwaite $\mathrm{N}$, Leech $\mathrm{S}$, et al. $\mathrm{BSACl}$ guideline: prescribing an adrenaline auto-injector. Clin Exp Allergy 2016;46(10):1258-1280. DOI: 10.1111/cea.12788.

7. Matsuura $\mathrm{H}$. Analysis of systemic complications and deaths during dental treatment in Japan. Anes Prog 1989;36(4-5):223-225.

8. Jevon P. Management of oxygen therapy in the dental practice. $\mathrm{Br}$ Dent J 2019;226(8):569-573. DOI: 10.1038/s41415-019-0200-3.

9. Coelho C, Mead M. Sepsis: the applicability to dental care professionals. Br Dent J 2018;225(12):1078-1081. DOI: 10.1038/ sj.bdj.2018.1039.

10. Jevon P. Basic guide to medical emergencies in the dental practice. 3rd ed. Oxford: Wiley Blackwell; 2021 [In Press].

11. Sdcep. Drug prescribing for dentistry: dental clinical guidance. 3rd ed. 2016. Available at: https:// www.sdcep.org.uk/wp-content/ uploads/2016/03/sdcep-drug-prescribing-for-dentistry-3rd-edition. pdf [Accessed June 2020].

12. Care Quality Commission. Dental mythbuster 9: hand hygiene. 2018. Available at: https://www.cqc.org.uk/guidance-providers/dentists/ dental-mythbuster-9-hand-hygiene.

13. Haas DA. Emergency drugs. Dent Clin North Am 2002;46(4):815-830. DOI: 10.1016/s0011-8532(02)00027-7.

14. Scully C, Cawson RA. Medical problems in dentistry. 5th ed. 2005.

15. Gilway $D$, Brown SJ. Medical emergencies: sepsis in primary dental care. Br Dent J 2016;220(6):278. DOI: 10.1038/sj.bdj. 2016.202.

16. Kasper DL, Braunwald E, Fauci AS, et al. Harrison's principles of internal medicine. 16th ed. 2005.

17. Travers A, Taylor K. Adrenaline use: the use of pre-filled adrenaline syringes in anaphylaxis kits. Br Dent J 2019;226(2):85-86. DOI: 10.1038/ sj.bdj.2019.57.

18. American Heart Association. In: Cummins RO, editor. Advanced cardiac life support provider manual. 2001.

19. Malamed SF. Medical emergencies in the dental office. 5th ed. St Louis: Mosby; 2000. p. 58-91.

20. Burkit's textbook of oral medicine. 11th ed. 2008.

21. Collange $O$, Bildstein A, Samin J, et al. Prevalence of medical emergencies in the dental practice. Resuscitation 2010;81(7):915-916. DOI: 10.1016/j.resuscitation.2010.03.039.

22. Fast TB, Martin MD, Ellis TM. Emergency preparedness: a survey of dental practitioners. J Am Dent Assoc 1986;112(4):499-501. DOI: 10.14219/jada.archive.1986.0043. 